\title{
Anti-interleukin-5 therapy in severe asthma
}

\author{
Gilles Garcia ${ }^{1,2,3}$, Camille Taillé ${ }^{4}$, Pierantonio Laveneziana ${ }^{1,2,3}$, Arnaud Bourdinn ${ }^{5,6}$, \\ Pascal Chanez ${ }^{7}$ and Marc Humbert ${ }^{1,2,3}$
}

\begin{abstract}
Affiliations: ${ }^{1}$ Faculté de médecine, Université Paris-Sud, Le Kremlin-Bicêtre, ${ }^{2}$ INSERM U999, LabEx LERMIT, Centre Chirurgical Marie Lannelongue, Le Plessis-Robinson, ${ }^{3}$ Service de Pneumologie. Assistance Publique Hôpitaux de Paris, DHU Thorax Innovation, Centre de Référence de l'Hypertension Pulmonaire Sévère, Hôpital Universitaire de Bicêtre, Le Kremlin-Bicêtre, ${ }^{4}$ Service de Pneumologie et Centre de Compétence des Maladies Pulmonaires Rares, Université Paris Diderot, Assistance Publique-Hôpitaux de Paris, Hôpital Bichat, INSERM U700, Paris, ${ }^{5}$ Dept of Respiratory Disease, Hôpital Arnaud de Villeneuve, CHU Montpellier, Montpellier, ${ }^{6}$ INSERM U1046 Physiologie et Médecine expérimentale du coeur et des muscles, Université Montpellier, CHU Arnaud de Villeneuve, Montpellier, and ${ }^{7}$ Département des Maladies Respiratoires, AP-HM, Laboratoire d'immunologie INSERM CNRS U 600, UMR6212, Aix Marseille Université, Marseille, France.
\end{abstract}

Correspondence: G. Garcia, Service de Pneumologie, Centre de Référence de l'Hypertension Pulmonaire Sévère, Hôpital Universitaire de Bicêtre, 78 Rue du Général Leclerc, 94270 Le Kremlin-Bicêtre, France. E-mail: gilles.garciaabct.aphp.fr

ABSTRACT Asthma is a chronic inflammatory disorder of the airways that leads to acute symptoms, exacerbations and sometimes, for a small part of the asthmatic population, fatal or near-fatal exacerbations. This as yet significant minority of individuals present with severe asthma and have persisting daily symptoms, and exacerbations despite compliance with high doses of inhaled steroids and additional treatment. For more than a decade, the pharmacological management of patients with severe asthma has focused on evaluating specific cytokines. The rationale of this approach is based on the distinguished key role played by eosinophils in the asthma inflammatory processes. Eosinophils are recruited from the circulation to airways where they cause airway damage via different mechanisms. Eosinophils are regulated in terms of their recruitment, activation, growth, differentiation and survival by interleukin (IL)-5. Abundant data from in vitro experiments, animal models and clinical trials has confirmed that IL-5 inhibition may be an effective approach for the treatment of asthma, especially severe asthma. Interfering with eosinophil function or reducing their numbers has been one of the most important goals of therapeutic monoclonal antibodies, which target cytokine receptor interactions in asthma, particularly IL-5. This review will consider new treatments options for severe asthma, particularly those targeting IL-5, that have already been evaluated in clinical trials in asthmatic patients.

0 @ERSpublications

Rationale, recent advances and future developments of anti-IL-5 therapy in patients with severe asthma http://ow.ly/n3viv

\section{Introduction}

Asthma is a chronic inflammatory disease of the airways. There are many inflammatory pathways involved in asthma, but eosinophils play a critical role in the pathogenesis and severity of the disease [1]. Interleukin (IL) -5 has been recognised as the most specific cytokine in the eosinophil lineage $[2,3]$ and has been identified as the key common denominator in inflammatory pathways in asthma [4]. IL-5 plays a key role in eosinophil proliferation, differentiation, maturation, migration to tissue sites and survival, as well as prevention of eosinophil apoptosis [5-7]. IL-5 comprises of a functional site for binding to the specific

Received: May 212013 | Accepted after revision: July 102013

Conflict of interest: Disclosures can be found alongside the online version of this article at err.ersjournals.com

Provenance: Publication of this peer-reviewed article was supported by the World Scleroderma Foundation, Switzerland (principal sponsor, European Respiratory Review issue 129).

Copyright (CERS 2013 
receptor subunit, IL-5R $\alpha$, and a separate motif for binding to the signalling subunit, $\beta$-chain [8]. The IL-5R $\alpha$ subunit is specific only to IL-5 binding; the $\beta$-chain also binds the cytokines IL-3 and granulocyte-macrophage colony-stimulating factor via a shared extracellular domain [9]. There is considerable emerging evidence that differentiation of eosinophils also occurs within tissues undergoing an allergic response, such as the airways in atopic asthma, and not only in bone marrow [7]. Thus, in order to obtain the most clinical response in eosinophilic asthma, a systemic approach to anti-IL-5 therapy may be important. Eotaxin is an accompanying chemokine with a dose-dependent capacity to stimulate migration of eosinophil CD34 ${ }^{+}$progenitor cells [10]. Some observations also suggest that the eotaxin/CCR3 interaction, independently of IL-5, may be involved in the survival and function of eosinophils in the airways and in clinical symptoms [11]. This further suggests that independent interactions between eotaxin and its receptor, CCR3, may be important in driving tissue events in asthma [12]. This may partially explain the apparent disconnection between eosinophil targeting and clinical response in asthma. The role of eosinophils has been critically re-evaluated after a study in which an anti-IL-5 antibody was shown to decrease blood and sputum eosinophil levels, without having an effect on late asthmatic response or airway hyperesponsiveness (AHR) $[13,14]$. Awareness of these emerging interactions involving eotaxins and their receptors may be important in a therapeutic context of developing new therapeutic approaches targeting the IL-5 pathway.

In the lung, T-lymphocytes are the main source of IL-5 [15], but they do not express IL-5R. Eosinophils and basophils express IL-5R on their surface and also produce IL-5, contributing to the levels of this cytokine. Several lines of experimental evidence support the role of IL-5 in the pathogenesis of asthma. IL-5 mRNA is identified in increased quantities in bronchial biopsies taken from asthmatics compared with non-asthmatic controls [16]. IL-5 mRNA expression in the airways has been shown to correlate with the clinical severity of asthma [17]. Inhaled bronchial provocation with allergen gives rise to an increase in IL-5 mRNA in bronchoalveolar lavage from asthmatics patients [18]. Finally, inhalation of recombinant human IL-5 leads to an increase in eosinophilia in induced sputum and in AHR [19]. These data confirm that IL-5 is present in patients with asthma, making it a possible target for intervention.

Severe asthma is a difficult concept without a clear definition. Patients with severe asthma represent a small subset, between $5 \%$ and $10 \%$, of the asthmatic population who experience persisting daily symptoms and exacerbations, despite compliance with high doses of inhaled steroids in combination with additional treatment [20-22]. It follows that new therapeutic options are the major unmet need for severe asthmatics patients [21]. Defining severe asthma is quite a challenge because the clinical presentation, type of inflammatory process, comorbidities and adherence to therapy affect the severity of each individual patient with severe asthma. Efforts have been made to phenotype patients with asthma [23-26], and it is now accepted that asthma is not only one phenotype but rather a heterogeneous mixture of syndromes. This is also true for severe asthma. Thus, there is not one single therapeutic approach to improve outcomes in patients with severe asthma but several, which are directed at reducing airway smooth muscle, or airway inflammatory cell number and/or activity, or targeting specific airway effector mediators [27]. One of the most prominent and emerging phenotypes is characterised by eosinophilic airway inflammation despite the use of high-dose anti-inflammatory treatment.

Asthma is associated with tissue eosinophilia in $40-60 \%$ of patients [28], and the intensity of eosinophilia has been correlated with asthma severity [29]. Most severe asthma exacerbations are eosinophilic and a neutrophilic infiltrate is less frequently observed [30]. Several studies have demonstrated that the risk of severe asthma exacerbations can be reduced by administering asthma treatment that minimises airway eosinophils (measured in induced sputum) [31, 32]. Targeting IL-5 (or IL-5R) is, therefore, an appealing approach to the treatment of patients with asthma and may have a beneficial therapeutic effect by preventing tissue damage in patients whose asthma is related to eosinophils. Anti-IL-5 antibodies target eosinophils by binding to IL-5 and preventing the ligation to IL-5R $\alpha$. Two different humanised anti-IL-5 antibodies, mepolizumab and reslizumab, and one humanised antil-IL-5R antibody, benralizumab, have been investigated in clinical trials for severe asthma [33].

\section{Anti-IL-5 therapeutic approaches}

Numerous clinical trials involving monoclonal antibodies to IL-5 and IL-5R are currently in progress at various phases of clinical development. Table 1 summarises these studies and the various IL-5/IL-5R approaches [13, 34-42]. Although patients receiving these monoclonal antibodies have demonstrated consistent reductions in eosinophil numbers in blood and sputum, clinical responses have been quite variable $[43,44]$. 
TABLE 1 Major findings from the main clinical trials evaluating anti-interleukin (IL)-5 antibodies in asthma

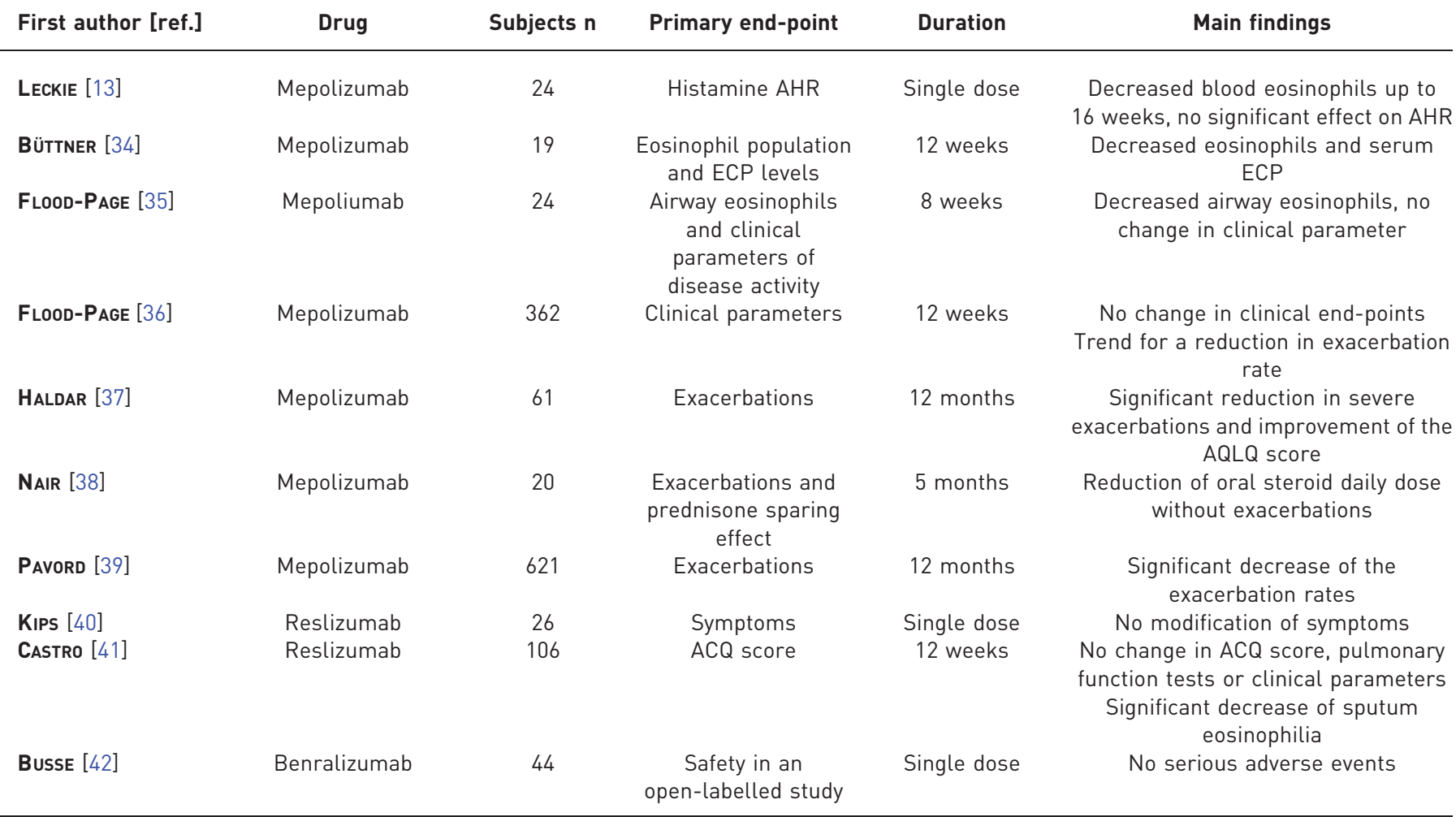

AHR: airway hyperresponsiveness; ECP: eosinophil cationic protein; $A C Q$ : asthma control questionnaire; $A Q L Q$ : Asthma Quality of Life Questionnaire.

\section{Mepolizumab}

Mepolizumab (SB 240563; GlaxoSmithKline, Research Triangle Park, NC, USA) is a humanised monoclonal antibody (IgG1) that binds with high affinity to free IL-5 and thus prevents IL-5 from binding to IL-5R $\alpha$. At present, mepolizumab is the most studied anti-IL-5 in the treatment of severe asthma. Mepolizumab has also been investigated for the treatment of atopic dermatitis, FIP1L1/PDGFRA-negative hypereosinophilic syndromes, eosinophilic oesophagitis, nasal polyposis and Churg-Strauss syndrome [45].

It has long been established that mepolizumab administered monthly via injections depletes white blood eosinophils and eosinophil cationic protein from the blood [34]. However, reductions in airway eosinophil counts are less clear, with results ranging from a 55\% decrease after 4 months of treatment to no significant change after 1 year of treatment $[35,37]$. This important decrease in the eosinophil count was also observed in the sputum of asthmatics patients. Disappointingly, despite this finding, the late asthmatic response to allergen challenge and AHR to inhaled histamine were not affected by mepolizumab [13, 36, 38].

The first large study of anti-IL-5 therapy in asthma investigated the effect of three intravenous infusions of mepolizumab (250 mg, $750 \mathrm{mg}$ or placebo) in 362 randomised patients with moderate asthma [36]. Both blood and sputum eosinophil counts were significantly decreased in patients receiving anti-IL-5. There were no statistically significant changes in any of the clinical end-points measured: morning asthma symptom scores, peak expiratory flow rate, forced expiratory volume in $1 \mathrm{~s}$ (FEV1), inhaled supplemental $\beta_{2}$-agonist use, or asthma-related quality of life scores. A nonsignificant trend for decreasing exacerbation rate in the group receiving mepolizumab $750 \mathrm{mg}$ was observed. These results were surprising because the observed severe decrease of blood and tissue eosinophilia was not sufficient to improve asthma symptoms and severity.

More recent studies have shown that mepolizumab reduces exacerbations in selected patients who have persistent sputum eosinophilia despite high doses of inhaled corticosteroids (ICS), although there is no improvement in symptoms, lung function or AHR [37, 38]. In the first study, HALDAR et al. [37] included severe refractory eosinophilic asthmatics with a persistent sputum eosinophilia (sputum 3\%). Patients were treated either with mepolizumab $(n=29 ; 750 \mathrm{mg}$ i.v. $)$ or placebo $(n=32)$ every month for 1 year. The mean number of exacerbations was reduced and asthma-related quality of life was significantly improved in the 
treated group. However, as with other studies evaluating mepolizumab, there were no significant differences between groups in terms of symptoms, FEV1 after bronchodilator administration, or AHR.

NAIR et al. [38] investigated the effect of monthly treatment with mepolizumab $750 \mathrm{mg}$ i.v. $(\mathrm{n}=9)$ or placebo $(n=11)$ for 4 months in patients with severe, oral corticosteroid-dependent asthma and sputum eosinophilia $(>3 \%)$. The authors showed that there was a significant reduction in the number of asthma exacerbations in patients treated with mepolizumab (one exacerbation) compared with those who received placebo ( 12 exacerbations in 10 patients; $\mathrm{p}<0.01$ ), and a significant increase in time to first exacerbation $(20$ weeks versus 12 weeks in the placebo group). There was also a significant reduction in prednisone dose in the mepolizumab group. Other important outcomes, such as FEV1 and Asthma Control Questionnaire (ACQ), were significantly improved from baseline while asthma symptoms were not. Despite subtle differences, these two proof-of-concept studies suggest that the blockade of IL-5 function may offer benefits as a steroid-sparing agent in reducing the frequency of severe exacerbations in severe refractory eosinophilic asthma.

The latest published study is a double-blind, placebo-controlled phase II study. PAVORD et al. [39] provided further convincing evidence for mepolizumab as an effective treatment in severe eosinophilic asthma. In this trial, 616 patients with severe asthma and evidence of eosinophilic inflammation were randomised to receive one of three doses of i.v. mepolizumab $(75 \mathrm{mg}, 250 \mathrm{mg}$ or $750 \mathrm{mg}$ ) or matched placebo every 4 weeks for 12 months. Compared with placebo, mepolizumab reduced the rate of clinically significant asthma exacerbations, which was set as the primary outcome measure $(48 \%, 39 \%$ and $52 \%$ reduction with $75 \mathrm{mg}$, $250 \mathrm{mg}$ and $750 \mathrm{mg}$, respectively). Visits to emergency departments and admissions also fell, although with varying statistical support.

\section{Reslizumab}

Reslizumab (SCH55700; Teva Pharmaceuticals, Petah Tikva, Israel) is a humanised IL-5 monoclonal antibody that has been previously investigated in the treatment of nasal polyps and is currently in clinical development for the treatment of asthma [46]. Reslizumab administered over 12 weeks failed to improve asthma control in 18 patients with severe uncontrolled asthma and sputum eosinophilia compared to eight patients who served as controls [40]. There was a reduction in the circulating eosinophil count and a significant, but transient, improvement in FEV1 [40]. A 15-week phase II study in patients with poorly controlled eosinophilic asthma (high doses of ICS and sputum eosinophils $>3 \%$ ) randomly assigned to receive treatment with reslizumab $3.0 \mathrm{mg} \cdot \mathrm{kg}^{-1}(\mathrm{n}=53)$ or placebo $(\mathrm{n}=53)$ showed significant reductions in sputum eosinophils, indicating that reslizumab was biologically active [41]. The ACQ score, which was the primary end-point, demonstrated a trend toward a significant improvement in treated patients. The ACQ improvement was significantly better in patients with nasal polyps who received reslizumab. The improvement in FEV1 was also statistically significant and clinically relevant in patients treated with antiIL-5. A trend, although statistically nonsignificant, toward a reduction in asthma exacerbations was also observed [41]. These results suggest that patients with nasal polyps may benefit more from reslizumab. These preliminary findings require confirmation in a larger study. To date, these are the only clinical studies published on the use of reslizumab in asthma.

\section{Benralizumab}

Benralizumab (MEDI-563; Kyowa Hakko Kirin/AstraZeneca) is a humanised anti-IL-5R $\alpha$ monoclonal antibody [42]. Benralizumab targets the effector cells that are driven by IL-5 (eosinophils/basophils) rather than the numerous cells that only produce IL-5. In vitro experiments demonstrated that benralizumab directly targets eosinophils for antibody-dependent cell-mediated cytotoxicity [47]. A single dose of benralizumab administered intravenously to 44 subjects with mild atopic asthma has been shown to induce dose-dependent peripheral blood eosinophil count depletion for at least 8-12 weeks [47]. Preliminary data in a phase II study has demonstrated biological activity in airway mucosa in eosinophilic asthmatic patients using ICS. Large clinical trials with this agent are currently awaited [48, 49].

\section{New approaches}

Recently, a new approach has been under investigation in severe asthma which used antisense oligonucleotide therapy to inhibit cytokine activity [50]. Antisense oligonucleotides consist of strands of nucleic acids that bind to complimentary mRNA. The aim is to reduce the levels of mRNA by preventing the specific gene transcription needed for cytokine receptor production. TPI ASM-8, an antisense oligonucleotide, targets against CCR3, which mediates the effect of some eosinophil chemoattractant [51]. TPI ASM- 8 has been shown to significantly reduce allergen airway eosinophilia in 14 stable, mild allergic asthmatics in a dose-dependent manner $[52,53]$. Doubling the dose of TPI ASM-8 reduced post-allergen AHR to methacholine [53]. Another approach is to use small interfering (si)RNA molecules against IL-5 or 
eotaxin to prevent protein transcription. Unfortunately, this approach is only limited to a murine model of asthma $[54,55]$.

It is now clear that anti-IL-5 therapies are biologically active and significantly reduce blood and sputum eosinophils and symptomatic exacerbations in patients with severe asthma. It is a promising approach that is currently under investigation in patients with severe asthma and persisting airway eosinophilia. However, several questions remain unanswered. In the DREAM (Dose Ranging Efficacy and Safety with Mepolizumab in Severe Asthma) study, there is dissociation between a significant reduction of exacerbation risk without any improvement on traditional markers of asthma control (FEV1, AQLQ and ACQ scores) [39]. This dissociation perhaps suggests that clinical parameters and exacerbations represent different aspects of the disease. In two studies assessing omalizumab efficacy, the rate of exacerbation was also significantly decreased with only a small improvement in the AQLQ scores $[56,57]$. The total asthma symptom scores, especially built for these studies, were improved but were difficult to compare against the results of the ACQ score used in the DREAM study. Concerning lebrikizumab (anti-IL-13 antibody) or dupilumab (anti-IL- $4 \alpha$ receptor antibody) there are only two proofs of concept studies which do not allow conclusion about potential dissociation of the efficacy (exacerbation versus symptom score) [58, 59]. In the DREAM study, the overall frequency of serious adverse events was similar between groups. Nonetheless, three patients died during the study in the treated group (acute pancreatitis and septic shock, suicide and acute asthma), but there is no evidence of a relationship between these deaths and the product under examination.

For daily clinical practice the major question is to better identify the best candidates to anti-IL-5 therapy. The good candidates appear to be the more severe asthmatic patients who do not achieve asthma control even with high doses of ICS in combination with additional treatment. Currently, for these patients the Global Initiative for asthma propose to use step 5 of the therapy guidelines and to use low doses of daily oral steroids or omalizumab. Omalizumab is indicated to allergic severe asthmatics patients and there are only few data concerning omalizumab in non-allergic severe asthma $[60,61]$. This also raises the question of the use of biomarkers that are able to identify possible responders. It is essential to promote the use of biomarkers in clinical studies of new severe asthma therapies and ultimately to validate them in routine clinical care. The major purpose of these biomarkers is to tailor the treatment approach by predicting responses to therapy. The potential role of periostin as a biomarker was recently noted [58]. HANANIA et al. [56] also suggests that the efficacy of omalizumab was greater in a subgroup of patients with higher biological levels of the following biomarkers: exhaled nitric oxide fraction, eosinophil blood count and periostin level. The ideal biomarker must be noninvasive, cheap, specific and reliable to predict the good clinical response, but also easily available. In previous studies, refractory eosinophilic asthma was defined on the basis of $\geqslant 3 \%$ eosinophils in induced sputum [37, 38]; however, in the DREAM study, the eosinophilic profile criteria was more available in daily clinical practice, such as blood eosinophil counts $>0.3 \times 10$ ? cells $\cdot \mathrm{L}^{-1}$ or exhaled nitric oxide levels $>50 \mathrm{ppb}$, and totally independent of the allergic status [39]. Currently, it seems that these biomarkers are biomarkers of an inflammatory T-helper-2 profile rather than a specific biomarker for one biotherapy. But some studies are needed to clarify the potential role of these biomarkers.

In conclusion, it is not easy to categorise severe asthmatics into endotypes or clinical subphenotypes because phenotypes may be overlapping in specific patients and may change over time. It is also not easy to select the right treatment for the right patient. Candidates for anti-IL-5 therapy seem to be severe eosinophilic asthmatic patients with a history of frequent exacerbations but this clinical profile must be confirmed.

\section{References}

Kazani S, Israel E. Update in asthma 2011. Am J Respir Crit Care Med 2012; 186: 35-40.

Sanderson CJ. Interleukin-5, eosinophils, and disease. Blood 1992; 79: 3101-3109.

Lee JJ, McGarry MP, Farmer SC, et al. Interleukin-5 expression in the lung epithelium of transgenic mice leads to pulmonary changes pathognomonic of asthma. J Exp Med 1997; 185: 2143-2156.

4 Inman MD. Bone marrow events in animal models of allergic inflammation and hyperresponsiveness. J Allergy Clin Immunol 2000; 106: Suppl. 5, S235-S241.

5 Sitkauskiene B, Johansson AK, Sergejeva S, et al. Regulation of bone marrow and airway CD34+ eosinophils by interleukin-5. Am J Respir Cell Mol Biol 2004; 30: 367-378.

6 Menzies-Gow A, Ying S, Phipps S, et al. Interactions between eotaxin, histamine and mast cells in early microvascular events associated with eosinophil recruitment to the site of allergic skin reactions in humans. Clin Exp Allergy 2004; 34: 1276-1282.

7 Rosenberg HF, Phipps S, Foster PS. Eosinophil trafficking in allergy and asthma. J Allergy Clin Immunol 2007; 119: $1303-1310$.

8 Molfino NA, Gossage D, Kolbeck R, et al. Molecular and clinical rationale for therapeutic targeting of interleukin-5 and its receptor. Clin Exp Allergy 2012; 42: 712-737.

9 Rossjohn J, McKinstry WJ, Woodcock JM, et al. Structure of the activation domain of the GM-CSF/IL-3/IL-5 receptor common beta-chain bound to an antagonist. Blood 2000; 95: 2491-2498. 
10 Sehmi R, Dorman S, Baatjes A, et al. Allergen-induced fluctuation in CC chemokine receptor 3 expression on bone marrow CD34+ cells from asthmatic subjects: significance for mobilization of haemopoietic progenitor cells in allergic inflammation. Immunology 2003; 109: 536-546.

11 Lamkhioued B, Abdelilah SG, Hamid Q, et al. The CCR3 receptor is involved in eosinophil differentiation and is up-regulated by Th2 cytokines in CD34+ progenitor cells. J Immunol 2003; 170: 537-547.

12 Park SW, Kim DJ, Chang HS, et al. Association of interleukin-5 and eotaxin with acute exacerbation of asthma. Int Arch Allergy Immunol 2003; 131: 283-290.

13 Leckie MJ, ten Brinke A, Khan J, et al. Effects of an interleukin-5 blocking monoclonal antibody on eosinophils, airway hyper-responsiveness, and the late asthmatic response. Lancet 2000; 356: 2144-2148.

14 Leckie MJ. Anti-interleukin-5 monoclonal antibodies: preclinical and clinical evidence in asthma models. Am J Respir Med 2003; 2: 245-259.

15 Ying S, Durham SR, Corrigan CJ, et al. Phenotype of cells expressing mRNA for TH2-type (interleukin 4 and interleukin 5) and TH1-type (interleukin 2 and interferon gamma) cytokines in bronchoalveolar lavage and bronchial biopsies from atopic asthmatic and normal control subjects. Am J Respir Cell Mol Biol 1995; 12: 477-487. Hamid Q, Azzawi M, Ying S, et al. Expression of mRNA for interleukin-5 in mucosal bronchial biopsies from asthma. J Clin Invest 1991; 87: 1541-1546.

17 Humbert M, Corrigan CJ, Kimmitt P, et al. Relationship between IL-4 and IL-5 mRNA expression and disease severity in atopic asthma. Am J Respir Crit Care Med 1997; 156: 704-708.

18 Robinson D, Hamid Q, Bentley A, et al. Activation of CD4+ T cells, increased TH2-type cytokine mRNA expression, and eosinophil recruitment in bronchoalveolar lavage after allergen inhalation challenge in patients with atopic asthma. J Allergy Clin Immunol 1993; 92: 313-324.

19 Shi HZ, Xiao CQ, Zhong D, et al. Effect of inhaled interleukin-5 on airway hyperreactivity and eosinophilia in asthmatics. Am I Respir Crit Care Med 1998; 157: 204-209.

20 Proceedings of the ATS workshop on refractory asthma: current understanding, recommendations, and unanswered questions. American Thoracic Society. Am J Respir Crit Care Med 2000; 162: 2341-2351.

21 Chanez P, Wenzel SE, Anderson GP, et al. Severe asthma in adults: what are the important questions? J Allergy Clin Immunol 2007; 119: 1337-1348.

22 Bel EH, Sousa A, Fleming L, et al. Diagnosis and definition of severe refractory asthma: an international consensus statement from the Innovative Medicine Initiative (IMI). Thorax 2011; 66: 910-917.

23 Moore WC, Meyers DA, Wenzel SE, et al. Identification of asthma phenotypes using cluster analysis in the Severe Asthma Research Program. Am J Respir Crit Care Med 2010; 181: 315-323.

24 Moore WC, Bleecker ER, Curran-Everett D, et al. Characterization of the severe asthma phenotype by the National Heart, Lung, and Blood Institute's Severe Asthma Research Program. J Allergy Clin Immunol 2007; 119: 405-413.

25 Wenzel SE, Schwartz LB, Langmack EL, et al. Evidence that severe asthma can be divided pathologically into two inflammatory subtypes with distinct physiologic and clinical characteristics. Am J Respir Crit Care Med 1999; 160: 1001-1008.

26 Wenzel SE. Asthma: defining of the persistent adult phenotypes. Lancet 2006; 368: 804-813.

27 O’Byrne PM, Naji N, Gauvreau GM. Severe asthma: future treatments. Clin Exp Allergy 2012; 42: 706-711.

28 Zhang JY, Wenzel SE. Tissue and BAL based biomarkers in asthma. Immunol Allergy Clin North Am 2007; 27: 623-632.

29 Bousquet J, Chanez P, Lacoste IY, et al. Eosinophilic inflammation in asthma. N Engl J Med 1990; 323: 1033-1039.

30 Turner MO, Hussack P, Sears MR, et al. Exacerbations of asthma without sputum eosinophilia. Thorax 1995; 50: 1057-1061.

31 Jayaram L, Pizzichini MM, Cook RJ, et al. Determining asthma treatment by monitoring sputum cell counts: effect on exacerbations. Eur Respir J 2006; 27: 483-494.

32 Green RH, Brightling CE, McKenna S, et al. Asthma exacerbations and sputum eosinophil counts: a randomised controlled trial. Lancet 2002; 360: 1715-1721.

33 O'Byrne PM. Cytokines or their antagonists for the treatment of asthma. Chest 2006; 130: 244-250.

34 Büttner C, Lun A, Splettstoesser T, et al. Monoclonal anti-interleukin-5 treatment suppresses eosinophil but not T-cell functions. Eur Respir J 2003; 21: 799-803.

35 Flood-Page PT, Menzies-Gow AN, Kay AB, et al. Eosinophil's role remains uncertain as anti-interleukin-5 only partially depletes numbers in asthmatic airway. Am I Respir Crit Care Med 2003; 167: 199-204.

36 Flood-Page P, Swenson C, Faiferman I, et al. A study to evaluate safety and efficacy of mepolizumab in patients with moderate persistent asthma. Am J Respir Crit Care Med 2007; 176: 1062-1071.

37 Haldar P, Brightling CE, Hargadon B, et al. Mepolizumab and exacerbations of refractory eosinophilic asthma. N Engl J Med 2009; 360: 973-984.

38 Nair P, Pizzichini MM, Kjarsgaard M, et al. Mepolizumab for prednisone-dependent asthma with sputum eosinophilia. N Engl J Med 2009; 360: 985-993.

39 Pavord ID, Korn S, Howarth P, et al. Mepolizumab for severe eosinophilic asthma (DREAM): a multicentre, double-blind, placebo-controlled trial. Lancet 2012; 380: 651-6519.

40 Kips JC, O'Connor BJ, Langley SJ, et al. Effect of SCH55700, a humanized anti-human interleukin-5 antibody, in severe persistent asthma: a pilot study. Am J Respir Crit Care Med 2003; 167: 1655-1659.

41 Castro M, Mathur S, Hargreave F, et al. Reslizumab for poorly controlled, eosinophilic asthma: a randomized, placebo-controlled study. Am J Respir Crit Care Med 2011; 184: 1125-1132.

42 Busse WW, Katial R, Gossage D, et al. Safety profile, pharmacokinetics, and biologic activity of MEDI-563, an antiIL-5 receptor alpha antibody, in a phase I study of subjects with mild asthma. J Allergy Clin Immunol 2010; 125 : 1237-1244.

43 Walsh GM. Mepolizumab and eosinophil-mediated disease. Curr Med Chem 2009; 16: 4774-4778.

44 Walsh GM. Reslizumab, a humanized anti-IL-5 mAb for the treatment of eosinophil-mediated inflammatory conditions. Curr Opin Mol Ther 2009; 11: 329-336.

45 Wechsler ME, Fulkerson PC, Bochner BS, et al. Novel targeted therapies for eosinophilic disorders. J Allergy Clin Immunol 2012; 130: 563-571.

46 Gevaert P, Bachert C, Holtappels G, et al. Enhanced soluble interleukin-5 receptor alpha expression in nasal polyposis. Allergy 2003; 58: 371-379. 

antibody-dependent cell-mediated cytotoxicity function. J Allergy Clin Immunol 2010; 125: 1344-1353. receptor alpha monoclonal antibody. Am J Respir Crit Care Med 2012; 185: A3961.

49 Molfino NA, Nowak R, Silverman RA, et al. Reduction in the number and severity of exacerbations following acute severe asthma: results of a placebo-controlled, randomized clinical trial with benralizumab. Am J Respir Crit Care Med 2012; 185: A2753.

50 Allakhverdi Z, Allam M, Renzi PM. Inhibition of antigen-induced eosinophilia and airway hyperresponsiveness by antisense oligonucleotides directed against the common beta chain of IL-3, IL-5, GM-CSF receptors in a rat model of allergic asthma. Am J Respir Crit Care Med 2002; 165: 1015-1021.

51 Imaoka H, Campbell H, Babirad I, et al. TPI ASM8 reduces eosinophil progenitors in sputum after allergen challenge. Clin Exp Allergy 2011; 41: 1740-1746.

52 Gauvreau GM, Boulet LP, Cockcroft DW, et al. Antisense therapy against CCR3 and the common beta chain attenuates allergen-induced eosinophilic responses. Am J Respir Crit Care Med 2008; 177: 952-958.

53 Gauvreau GM, Pageau R, Séguin R, et al. Dose-response effects of TPI ASM8 in asthmatics after allergen. Allergy 2011; 66: 1242-1248.

54 Hammond SM, Caudy AA, Hannon GJ. Post-transcriptional gene silencing by double-stranded RNA. Nat Rev Genet 2001; 2: 110-119.

55 Huang HY, Lee CC, Chiang BL. Small interfering RNA against interleukin-5 decreases airway eosinophilia and hyper-responsiveness. Gene Ther 2008; 15: 660-667.

56 Hanania NA, Alpan O, Hamilos DL, et al. Omalizumab in severe allergic asthma inadequately controlled with standard therapy: a randomized trial. Ann Intern Med 2011; 154: 573-582.

57 Humbert M, Beasley R, Ayres J, et al. Benefits of omalizumab as add-on therapy in patients with severe persistent asthma who are inadequately controlled despite best available therapy (GINA 2002 step 4 treatment): INNOVATE. Allergy 2005; 60: 309-316.

58 Corren J, Lemanske RF, Hanania NA, et al. Lebrikizumab treatment in adults with asthma. N Engl J Med 2011; 365: 1088-1098.

59 Wenzel S, Ford L, Pearlman D, et al. Dupilumab in persistent asthma with elevated eosinophil levels. N Engl J Med 2013; 368: 2455-2466.

60 Garcia G, Magnan A, Chiron R, et al. A proof of concept randomized-controlled trial of omalizumab in patients with severe difficult to control nonatopic asthma. Chest 2013 [Epub ahead of print DOI: 10.1378/chest.12-1961].

1 de Llano LP, Vennera Mdel C, Álvarez FJ, et al. Effects of omalizumab in non-atopic asthma: results from a Spanish multicenter registry. J Asthma 2013; 50: 296-301. 